Beate Rosenzweig

\title{
Gleichheit, Differenz und Differenzen - Feministische Kontroversen um die politische Philosophie Platons ${ }^{1}$
}

"War Platon ein Feminist?" (vgl. Benhabib/Nicholson 1987, 513). Diese zunächst höchst erstaunlich anmutende Frage löste im Zuge der feministischen Auseinandersetzung über die politische Philosophie Platons und das darin vertretene $\mathrm{Ge}-$ schlechterverständnis eine lebhafte Kontroverse aus, die bis heute andauert. Die Grundlage für diese Diskussion bilden Platons Vorstellungen von einer Frauenund Kindergemeinschaft, wie er sie im 5. Buch seines staatsphilosophischen Hauptwerkes Politeia darlegt.

Es gibt also, mein Freund, keine die Staatsverwaltung betreffende Beschäftigung, die der Frau als Frau oder dem Mann als Mann zukäme; vielmehr sind die natürlichen Anlagen gleichmäßig unter beiden Geschlechtern verteilt, und naturgemäß hat die Frau ebenso wie der Mann Anspruch auf alle Beschäftigungen, bei allen aber ist die Frau schwächer als der Mann. (Platon, Politeia 455d-e)

In seinem Gedankenentwurf einer Gemeinschaft von Wächtern und Wächterinnen fordert er das Recht auf gleiche Erziehung, das Recht auf einen ungehinderten sozialen Umgang, das Recht auf Berufsausübung, konstatiert die Rechtsfähigkeit von Frauen und - damit nicht genug - auch das Recht auf die freie Partnerwahl. Diese Gleichheitsforderungen basieren allerdings, wie das Einstiegszitat bereits deutlich macht, auf der Annahme einer generellen körperlich bedingten Unterlegenheit von Frauen. Die Frage, wie die ideengeschichtliche Einmaligkeit des platonischen Gedankenexperimentes zu erklären und das Verhältnis von Geschlechtergleichheit und -differenz zu deuten ist, ist bis heute konstitutiv für feministische Platonanalysen.

Wie Natalie Harris Bluestone in ihrem grundlegenden und für die feministische Klassikerforschung einzigartigen Band über die Rezeptionsgeschichte des platonischen Frauenbildes fundiert nachweist, bildeten feministische Forschungen seit Anfang der 70er Jahre einen Wendepunkt innerhalb der Platonforschung. Waren bis dahin Platons Vorstellungen zur Geschlechtergleichheit von der Forschung entweder gänzlich ignoriert oder unter dem Stichwort "nicht ernstzunehmend" ad acta gelegt worden, so entwickelte sich erst im Zuge der zweiten Frauenbewegung eine tiefer gehende Analyse dieser Textpassagen (Bluestone 1987).

1 Ich möchte mich bei den beiden anonymen Gutachterinnen der Feministischen Studien herzlich für ihre hilfreichen Kommentare bedanken. 
Seitdem erfreut sich die politische Philosophie Platons einer feministischen Dauerkonjunktur. Quantitativ gesehen lassen sich drei Hochphasen der Rezeption ausmachen, die erste von Anfang bis Mitte der siebziger Jahre, dann erneut ab Mitte der achtziger Jahre und eine jüngste Neuauflage der Debatten seit Mitte der 90er Jahre. Letztere ist $\mathrm{zu}$ großen Teilen gespeist aus aktualisierten Kommentierungen bereits veröffentlichter Studien.

Platons Werk, respektive seine staatsphilosophischen Hauptwerke Politeia und Nomoi, gehören damit zu den wohl meistgelesenen Werken der politischen Philosophiegeschichte - das Whiteheadsche Diktum von der gesamten abendländischen Philosophie als einer Reihe von Fußnoten zu Platon ist legendär - und seine Gedanken haben feministische AutorInnen fortgesetzt zu immer neuen Analysen herausgefordert. Die Ergebnisse und Kontroversen feministischer Platonanalysen sind allerdings - wie die Ergebnisse feministischer Ideengeschichtsanalysen insgesamt - bis heute kaum in den fachwissenschaftlichen mainstream integriert. Allenfalls wird eine Autorin/eine These als repräsentativ für den feministischen Blick auf die politische Philosophie Platons herausgehoben (vgl. u. a. Höffe 1997, Zehnpfennig 1997, Kersting 1999, Ottmann 2001). Der folgende Beitrag sucht dieser bis heute vorherrschenden, vereinheitlichenden Sicht auf feministische Ideengeschichtsforschungen insofern entgegenzuwirken als am Beispiel Platons im Folgenden die wichtigsten Etappen feministischer Rezeption herausgearbeitet und analysiert werden. Hierbei werden die Gemeinsamkeiten und Unterschiede der Platonrezeptionen im Zentrum stehen. Am Beispiel der politischen Philosophie Platons lässt sich meines Erachtens eindrücklich die Pluralität feministischer Ideengeschichtsforschungen deutlich machen. Feministische politische Ideengeschichtsschreibung erweist sich nicht nur, wie Herfried Münkler grundsätzlich formuliert, als ein »Ort fortwährenden Ausprobierens und Experimentierens" (Münkler 2003, 103), sondern vor allem auch als ein Schauplatz andauernder theoretischer Selbstreflexionen.

\section{Platon - ein "profeministischer Klassiker" gleichheitstheoretisch gedeutet}

Auf der Grundlage gleichheitstheoretischer Annahmen zeichneten Autorinnen wie Abigail Rosenthal, Martha Lee Osborne, Christine Allen und Dorothea Wender zunächst ein profeministisches Platonbild, in dem alle werk- und textimmanenten Widersprüche unter Betonung der Einmaligkeit und Besonderheit Platons ignoriert, geglättet oder zumindest deutlich abgeschwächt wurden. Platons "Feminismus" erschien als eine einzigartige Ausnahme in der Geschichte der politischen Philosophie (Rosenthal 1973, 32), als Beleg für die Irrelevanz biologischer Geschlechtsunterschiede (Osborne 1975) und als grundlegendes 
Beispiel für eine Politik der Geschlechtergleichheit. Erste Gegenthesen, wie beispielsweise die von Sarah B. Pomeroy, selbst Platon sei nicht in der Lage gewesen, die Gleichheit der Geschlechter zu denken, werden schnell als Ausdruck »mangelnder Fairness" gewertet (Pomeroy 1974, Fortenbaugh 1975). ${ }^{2}$

Rekurrierend auf eine breiter angelegte Platonlektüre (Politeia, Nomoi, Timaeus, Symposion, Phaedrus) bescheinigt beispielsweise Christine G. Allen Platon, was die Gleichheit der Frauen angeht, durchgängige theoretische Konsistenz (Allen 1975, 131ff, vgl. auch Pierce 1973, 10f.). Ihre Argumentation stützt sich dabei fast ausschließlich auf die platonische Seelenlehre, die grundsätzliche Seelengleichheit von Mann und Frau und die Unsterblichkeit der Seele. Deutlich fundierter als Allen, allerdings zu ganz ähnlichen Schlussfolgerungen kommend, setzt sich Dorothea Wender mit den von ihr als "delightfully radical" bezeichneten Vorschlägen Platons zur Geschlechtergleichheit auseinander (Wender 1973, 76). Schon im Titel ihrer Analyse verweist sie auf die textimmanenten Widersprüche in Platons Werken und die Schwierigkeit einer Einordnung: "Misogynist, Paedophile, and Feminist."Wender hält Platon dennoch für kompatibel mit ihrer - nicht nur aus heutiger Sicht ungemein konturlosen und oberflächlich anmutenden - Definition von Feminismus: "When I say "feminist", I mean a man or a woman who believes that women should be given a better place in society (legally, politically, professionally, etc.) or one which more closely approximates that held by men of the same class." (Wender 1973, 75). Platons Forderungen nach einer völligen Chancengleichheit von Frauen und Männern reichten darüber hinaus, was die Abschaffung aller häuslichen Tätigkeiten für Frauen anginge, weiter als die vieler Feministinnen "heutzutage «. Nachweise für Platons Feminismus findet Wender nicht nur in der Politeia, sondern auch in den Nomoi, in denen Platons feministisches Programm etwas abgeschwächt, aber dennoch am Prinzip der Geschlechtergleichheit festgehalten werde. Die in Platons Werken gleichzeitig vorhandenen misogynen Äußerungen erklärt Wender indes aus der inneren Widersprüchlichkeit des Autors selbst ${ }^{3}$ : "The apparent paradox should now be clear: Plato both despised women as a class, and advocated more liberalism and privilege for them than any man in history had ever done, so far as we know. If I may borrow Patin's marvellous mot about Lucretius, we have here a striking example of l'Anti-Platon chez Platon. (Wender 1973, 82) Platon, resp. sein sprechendes alter ego Sokrates, habe die Frauen zwar nicht gemocht oder

2 Pomeroys These lautet, dass die Frauen im Wächterstand als Eigentum der Männer aufgefasst werden müssen und leitet dies aus dem Begriff koinonia ab. Als direkte Replik auf die These von Sarah B. Pomeroy, Platon sei "uncapable of seeing women as equal to men" und habe die Frauen im Wächterstand als Gemeinbesitz der männlichen Wächter gesehen, formulierte W.W. Fortenbaugh: "All this seems to me more than a bit unfair to Plato. "Fortenbaugh spricht demgegenüber davon, dass die weiblichen Wächterinnen als ebenbürtige Partnerinnen der Wächter aufgefasst werden müssten.Vgl. Pomeroy 1974, 33f.; Fortenbaugh 1975, 1.

3 Als Belege für die in der Politeia zum Ausdruck kommende Misogynität Platons führt Wender unter anderem folgende Textbeispiele an: 395d-e, 549c-d. 
gar bewundert, wie Wender einschränkend hervorhebt, er habe aber immerhin das Gefuihl gehabt, es sei gerecht und "zudem auch nur nützlich "uns eine Chance zu geben" (Wender 1973, 90). Wenders Argumentation erscheint mir nicht nur aufgrund ihres amorphen Feminismusbegriffes, sondern auch aufgrund der von ihr dezidiert vollzogenen Relation zwischen platonischem Feminismus und Homosexualität grundlegend zu kritisieren. Ihre Begründung für einen platonischen Feminismus beruht auf der Annahme, dass sowohl Platons profeministische Ideen als auch seine Misogynität aus seinen homosexuellen Präferenzen zu erklären sind. ${ }^{4}$ "If he dislikes women as he finds them, he is willing to try changing them" (Wender 1973, 88f.). Die Widersprüche in Platons Werk werden bei Wender ausdrücklich durch den Verweis auf die in sich widersprüchliche Haltung eines homosexuellen Mannes geklärt, dessen Frauenfeindlichkeit oder zumindest ausgeprägtes Desinteresse an den Frauen letztlich das Tor zur Emanzipation der Frauen aufstößt. So reflektiert Wender zwar deutlicher als die zuvor besprochenen Autorinnen die Widersprüche in Platons Werk, glättet diese aber mit Hilfe der Konstruktion einer binären Identitätslogik zwischen heterosexueller und homosexueller Männlichkeit. Während erstere aufgrund der Zuneigung zum weiblichen Geschlecht und der Furcht vor der weiblichen Macht rein an der konservativen Aufrechterhaltung der herrschenden Geschlechterverhältnisse interessiert sei, entfalte Letztere zumindest auf gedanklicher Ebene transformatives Potenzial. Dieses gründet, folgt man Wender, auf der Ablehnung heterosexuellen, körperlichen Begehrens und dem letztlich aus einer misogynen Grundhaltung entstandenen Wunsch nach einer Veränderung der "natürlichen" Geschlechterordnung. Für Wenders profeministische Einordnung Platons, die im Übrigen von Natalie Bluestone als "informativ und amüsant« bezeichnet wurde (Bluestone 1987, 87), bedarf es somit einer unter feministischen Vorzeichen vollzogenen Gleichsetzung von Misogynität und Homosexualität. Dieser von Wender letztlich profeministisch gedeutete Zusammenhang sollte auch in der weiteren Platonrezeption noch eine Rolle spielen.

Erst Mitte der neunziger Jahre hat Christine Pierce, bezogen auf die Forschungen von Susan Moller Okin und Jean Bethke Elshtain, erstmals auf diesen bias - die Abwertung der Homosexualität - in der feministischen Platonrezeption hingewiesen (Pierce 1994, 25ff). ${ }^{5}$ Wender ist damit nur ein Beispiel, dem andere, allerdings zur Untermauerung der platonischen Misogynität, folgten.

4 Eine These, die im übrigen, wie Arlene Saxonhouse zeigt, erstmals von Hans Kelsen (1942) in seiner psychoanalytischen Studie über Platons sexuelle Orientierung vertreten worden ist. Vgl. Saxonhouse 1976, 210.

5 Für Christine Pierce sind die Forschungen von Okin und Elshtain von Ärger über den Ausschluss von Frauen aus der homoerotischen Atmosphäre in den platonischen Dialogen geprägt. Deshalb werde Platons Misogynität zumindest teilweise in Relation zu seiner Homosexualität gesehen. Pierce Kritik an Okin und Elshtain richtet sich darüber hinaus auch gegen die Gleichsetzung von Homosexualität mit männlicher Homosexualität. Vgl. Pierce 1994, S. 31. 
Obwohl sich gegen die These eines platonischen Feminismus schon kurze Zeit später heftiger feministischer Widerstand regte, kann sie als konstitutives Element feministischer Platonrezeption gelten. Die Mehrzahl der AutorInnen setzt sich bis heute zumindest ex negativo damit auseinander und sorgt damit ebenfalls für die feministische Tradierung. In nahezu allen Veröffentlichungen und Sammelbänden der achtziger und neunziger Jahre zu feministischen Analysen platonischer Texte dient die Profeminismus-These als Aufhänger, die Kontroverse um Platons Feminismus als Beleg für die Vielfalt und Bandbreite feministischer Platonrezeptionen (vgl. Tuana 1994, 3ff; Ward 1996, XVff., Kraut, 1997, XI). Darüber hinaus gibt es immer wieder neue Unterstützung für die Annahme, Platons Sichtweisen seien feministisch oder zumindest vereinbar mit feministischen Prinzipien (vgl. auch Rauschenbach 1998, 35). Ganz nach dem Motto des halbvollen oder halbleeren Wasserglases wird Platon aufgrund der ideengeschichtlichen Einmaligkeit seiner Vorschläge und vor dem Hintergrund der ansonsten vorherrschen Misogynität in der politischen Theorie fortgesetzt positiv feministisch gewendet. Seine Schriften werden als "first articulation of sex unity or unisex theory in the history of philosophy of woman and man " Prudence Allen 1987, 264), als revolutionär (Okin 1977, 11), ja sogar als Schritt in Richtung auf eine konstitutionelle Demokratie mit allgemeinem Wahlrecht (Cohen 1987) gewertet. Platon habe die Chancengleichheit der Geschlechter im Sinn gehabt (Martin 1985), argumentiere für die "uneingeschränkte Gleichstellung von Mann und Frau« (Heinz 2002) ${ }^{6}$ und habe die gesellschaftliche Konstruiertheit von Geschlechterrollen erkannt (J. F. Smith 1983).

Auffällig ist, dass der jeweils zugrunde gelegte Begriff des "Feminismus" dabei zunächst gleichheitstheoretisch ausgedeutet worden ist, die Argumente für die Profeminismus-These seit Mitte der achtziger Jahre auch differenztheoretisch fundiert werden. Ein intensiv rezipiertes jüngeres Beispiel für eine gleichheitstheoretische Lesart sind die Überlegungen von Gregory Vlastos "Was Plato a Feminist?" (1997). Als Maßstab für die profeministische Einordnung Platons schlägt Vlastos eine liberale Feminismusdefinition in Anlehnung an die amerikanische Verfassung vor: sequality in the rights of persons shall not be denied or abridged on account of sex“. Vor diesem Hintergrund könne Platon durchaus, was seine Vorschläge in der Politeia zur Stellung der Frauen im Wächterstand anginge, als "der erste Feminist" bezeichnet werden. Darüber hinaus seien Platons Vorstellungen in Bezug auf die Frauen aller anderen Stände seines Idealstaates als antifeministisch einzustufen, genauso wie seine persönliche Haltung gegenüber den Frauen innerhalb der athenischen Gesellschaft. Platons Verdienst sei es dem-

6 In der deutschsprachigen Literatur wird Platon in den wenigen, bisher vorhandenen Einführungsbänden zur politischen Philosophie unter Geschlechterperspektive weitgehend als Begründer der Geschlechtergleichheit interpretiert, vgl. Doyé/Heinz/Kuster 2002, Rauschenbach 1998, vgl. auch Heinz 1997, zur genaueren Analyse des Verhältnisses von Geschlechtergleichheit und -differenz bei Platon vgl. Föllinger 1996. 
zufolge für eine Elite von Frauen von seinen eigenen antifeministischen Vorurteilen abzusehen. "In a triumph of imaginative impartiality he separated the character his inherited prejudices imputed to the masses of women in his own society from the character which, he reasoned, a few exceptional women could develop under ideal conditions of equal nurture (...)«(Vlastos 1997, 127).Vlastos differenziert damit deutlich zwischen Frauen verschiedener sozialer Schichten in Platons Idealstaatsentwurf und plädiert insbesondere vor dem Hintergrund der historischen Stellung der athenischen Frauen für das Festhalten an der These eines - allerdings sozial stark eingeschränkten - platonischen Feminismus. ${ }^{7}$ In dieser Hinsicht unterscheidet sich Vlastos These deutlich von den anfänglich zitierten profeministischen Einordnungen von Pierce, Allen und auch Wender.

\section{Platons Profeminismus - differenztheoretische Umdeutung}

Aus differenztheoretischer Perspektive ordnet Monique Canto Platon aufgrund seines Umgangs mit der "weiblichen Andersheit« als feministisch ein. Anders als Vlastos bezieht sie sich dabei nicht auf die Politeia, sondern charakterisiert die Nomoi als ein "feminist political manifesto" (Canto 1986, 339). Feministische Politik definiert Canto über den Begriff der weiblichen Präsenz. Von feministischer Politik könne demnach nur dann die Rede sein, wif women together with their bodies, their work, their labor, and their voice, are present in a place where everyone can see them (...)" (Canto 1986, 339). Während Platon in seinem Idealstaatsentwurf in der Politeia die Unterschiede zwischen den Geschlechtern neutralisiert hätte, habe er in den Nomoi die weibliche Andersheit als Grundbedingung des Politischen anerkannt. Frauen repräsentierten das Prinzip der Reproduktion, des Begehrens, der Differenz (Canto 1986, 349). Cantos Platoninterpretation basiert auf der Grundannahme komplementärer, binärer Geschlechterdifferenz, ihr geht es um die Präsenz des weiblichen Prinzips, das sie mit reproduktiven Fähigkeiten, Emotionalität, Begehren gleichsetzt. Die Geschlechterdifferenz erscheint als Grundlage der politischen Ordnung, die Anerkennung der Differenz als Grundvoraussetzung feministischer Politik. »For the necessity of the other and the reality of becoming are preconditions for conceiving the human world, the sexual and political world, the world of men and women, of cities and images, the world, finally, that is forced to become more fully conscious of the conditions of its own existence because of women's political share and presence in this world." (Canto 1986, 352). In Cantos emphatischer Würdigung weiblicher Differenz bleibt kein Raum für die Differenzen zwischen Frauen und Männern, für soziale und politische (Status-)unterschiede jeglicher Art. Die politische

7 Ähnlich argumentiert auch Janet F. Smith, die herausstellt, dass Platon die individuellen Unterschiede zwischen Frauen als größer erachtet als die zwischen Frauen und Männern, vgl. dies. $1994,39 f$. 
Präsenz des Anderen, die Canto als feministische Vision vor Augen hat, gründet auf der Prämisse natürlicher, essentialistischer Geschlechtsidentitäten. Nicht die Gleichheit der Geschlechter, sondern die Anerkennung ihrer Differenz bildet damit den Maßstab für eine feministische Apostrophierung Platons. Der Preis, den Canto dafür zu zahlen bereit ist, ist hoch: er umfasst neben der Überbetonung naturalisierter weiblicher Werte die vollständige Ausblendung der Widersprüche in Platons Denken und seiner an zahlreichen Textstellen zu belegenden Misogynität.

Für die Untermauerung der These eines platonischen Feminismus bleibt die differenztheoretische Lesart Cantos jedenfalls, auch in Bezug auf die Betonung der herausragenden Stellung der Nomoi, eine Ausnahme. Insgesamt wird die Profeminismusthese nur von einer Minderheit der InterpretInnen vertreten. Die Argumente hierfür werden allerdings, so lässt sich festhalten, mehrheitlich im Rückgriff auf gleichheitstheoretische Argumente und unter besonderer Hervorhebung der Politeia vorgetragen. Für die seit Mitte der 70er Jahre zu findenden Gegenthesen zu einem "angeblichen Feminismus Platons" lässt sich demgegenüber in den achtziger Jahren von einem Gleichgewicht zwischen differenz- und gleichheitstheoretisch orientierten Interpretationsansätzen sprechen.(vgl. Föllinger 1996, 58). Gemeinsam ist diesen Ansätzen, dass sie den Begriff "feministisch" in Bezug auf Platons Überlegungen grundsätzlich zurückweisen und dementsprechend die These von Geschlechtergleichheit in Platons Werken ebenso verneinen wie die der Anerkennung weiblicher Differenz durch Platon.

\section{"Feministischer Platonmythos" - gleichheits- und differenztheoretische Relektüren}

"Platon the feminist is a myth " Mit diesen Worten beendet Julia Annas ihre Analyse zum Verhältnis von "Plato's Republic and Feminism" (Annas 1976, 12). Annas zufolge sind Platons Vorstellungen zur Frauen- und Kindergemeinschaft im Wächterstand weder mit liberalfeministischen Gleichheitsvorstellungen noch mit radikalfeministischen weiblichen Selbstverwirklichungsvorstellungen vereinbar. "His arguments are unacceptable to a feminist, and the proposals made in Republic V are irrelevant to contemporary debate." (Annas 1976, 307). Die Gleichstellung der Frauen im Wächterstand sei rein utilitaristisch als Mittel der Ressourcenausbeutung zum Wohle des durchweg autoritär angelegten Idealstaates $\mathrm{zu}$ verstehen. Platon habe keinerlei Interesse an der Befreiung der Frauen gehabt, die gleiche Erziehung von Männern und Frauen ziele ausschließlich auf die Herstellung größtmöglicher Einheit im Staat. Dies zeige sich auch an seinen Ausführungen zur grundsätzlichen Unterlegenheit der Frauen und die starke soziale Beschränkung der Gleichheitsvorstellungen rein auf den Wächterstand. "No modern feminist would argue that women should be able to do men's jobs 
when this will result in greater direct benefit to the state and otherwise stay at home." (Annas 1976, 315).

Annas Thesen von der sozialen Exklusivität der Gleichheitsvorstellungen für Frauen und Männer und ihres rein funktionalistischen Stellenwertes in Platons Werk erfuhren in einer Reihe weiterer Forschungen $u$. a. von Linda Lange und Susan Moller Okin Bestätigung - allerdings nicht immer, wie das Beispiel von Lange zeigt, mit dem wissenschaftlich zu erwartenden Nachweis ihrer Herkunft. So reproduziert Linda Lange in wesentlichen Teilen ihrer Argumentation die Thesen von Julia Annas, erwähnt die Autorin allerdings nur am Rande und vernachlässigt darüber hinaus auch die für ihre Ausführungen ganz wesentlichen Forschungen von Susan Pomeroy und W.W. Fortenbaugh (Lange 1979, 4ff). Ausgehend von der von Platon vertretenen hierarchischen Arbeitsteilung zwischen den verschiedenen Ständen und der Trennung zwischen Privatheit und Öffentlichkeit hält Lange eine profeministische Einordnung Platons für nicht begründbar. "His theoretical concerns are ultimately not those of feminism "(Lange 1979, 5). Für Lange macht Platons primäre Zielsetzung, die Stabilität und Einheit des Staates, eine Auflösung des Privaten im Wächterstand nötig. Das Private erscheine ihm als Quelle von Partikularität und Uneinigkeit und müsse deshalb zumindest, wie in den Nomoi ausgefuihrt, unter öffentliche Kontrolle gestellt werden. Platon sei nicht an der Partizipation von Frauen im Wächterstand interessiert.Vielmehr gehe er von einer de facto Korrelation von Weiblichkeit und Unterlegenheit aus. Seine Vorschläge für die Frauen im Wächterstand bedeuteten nicht, und hier geht Lange deutlich über die Argumentation von Annas hinaus, dass Frauen überhaupt Teil des Wächterstandes seien (Lange 1979, 4).Vielmehr müsse davon ausgegangen werden, dass Platon die Frauen im Wächterstand als Gemeinbesitz (koinonnia) der Männer gesehen habe und keineswegs als gleichrangige Partnerinnen. ${ }^{8}$ Für Lange erweist sich Platon insgesamt als ein sexistisch argumentierender Autor. Seine sprichwörtliche Besonderheit entpuppt sich demnach, folgt man ihrer Interpretation, bei genauerem Hinsehen als völlige Fehlperzeption einer konsistent gedachten, misogynen politischen Theorie (Lange 1979, 13). Das Ziel der staatlichen Einheit gründet Lange zufolge auf der Unterlegenheit und Unterordnung der Frauen in allen gesellschaftlichen Schichten. Damit lässt sie, um eines ungebrochenen Sexismusnachweises willen, das von Platon hervorgehobene Potenzial gleicher Fähigkeiten von Frauen und Männern gänzlich außer Acht (vgl. Bluestone 1987, 99).

Auch für die fast zeitgleich erschienenen Überlegungen von Susan Moller Okin bildete die Trennung zwischen Öffentlichkeit und Privatheit den Ausgangspunkt ihrer Platoninterpretation (Okin 1977). Okins Interpretation nimmt

8 Vgl. hierzu die Diskussion von Pomeroy und Fortenbaugh. Während der Begriff koinõnia von Pomeroy wie auch bei Lange als Gemeinbesitz gedeutet wird, betont Fortenbaugh, dass der Begriff mehrere Bedeutungen umfasst und auch als gemeinsam im Sinne von partnerschaftlich aufgefasst werden kann, vgl. auch Bluestone (1987), S. 93f. 
in der Hochphase der feministischen Diskussion um "Platons Feminismus" insofern eine Sonderstellung ein, als sie den Begriff "feministisch" in ihren Ausführungen an keiner Stelle erwähnt. Sie stellt keinerlei Bezüge zur schwelenden Debatte um "Platons Feminismus" her und verweist nur ganz allgemein auf die Vielzahl von Forschungen zum Thema Frauen in Platons Texten. Platons Vorstellungen in der Politeia erscheinen ihr im Vergleich zu denen anderer klassischer Autoren als revolutionär, nicht mit Bezug auf gegenwärtige Feminismusdebatten. Ihre Analyse mündet in die These von einer rein funktionalistischen Einfuihrung der Geschlechtergleichheit durch Platon in der Politeia. Den Grund hierfür sieht sie in der Auflösung der Privatfamilie. "I contend that when one compares the arguments and proposals of the Republic with those of the Laws, it becomes clear that the absence of the presence of private family determines whether Plato advocates putting into practice his increasingly radical beliefs about the potential of women." (Okin 1992,11). Für Okin besteht demnach ein Kausalzusammenhang zwischen der Abschaffung von Privateigentum und Familie einerseits und der Gleichstellung von Frauen andererseits. Sie lehnt sich hiermit explizit an eine schon von Jean Jacques Rousseau in seinem Erziehungsroman Emile formulierte Erklärung an: "Da er [Platon] in seinem Staat die Einzelfamilie abschaffte und nicht mehr wusste, was er mit den Frauen machen sollte, machte er gezwungenermaßen Männer aus ihnen." (Rousseau 1993, 391). Die Abschaffung der Privatfamilie bedingt, soweit folgt Okin Rousseau, eine veränderte Stellung der Frauen. Allerdings bedeute dies nicht die von Rousseau nahe gelegte "Vermännlichung" der Frauen, sondern aus der inneren Logik des Platonischen Gedankenexperimentes folge die Gleichstellung der Frauen im Wächterstand. "If the female guardians were no longer to be defined in relation to particular men, children, and households, it seems that Plato had no alternative but to consider them persons in their own right." (Okin 1992, 19). Platons Bedeutung liegt Okin zufolge darin, dass er in der Lage gewesen sei, den Zusammenhang zwischen biologischer Geschlechterdifferenz und konventioneller Rollenzuweisung gedanklich aufzulösen und das natürliche Potenzial von Frauen für die politische Ordnung zu erkennen. Hieraus erklärten sich auch die Unterschiede zwischen der Politeia und den Nomoi. Mit der Wiedereinführung des Privateigentums und der Familie im zweitbesten Staat wird die Gleichheit zwischen Frauen und Männern zurückgenommen, auch wenn, wie Okin konzediert, Platon den Frauen selbst dann noch einen besseren Status zugesteht, als dies die zeithistorischen Umstände nahe legten. Privateigentum, Privatfamilie und die geschlechtspezifische Trennung von Privatheit und Öffentlichkeit be-

9 Während Okin in einer ersten Version ihrer Thesen davon ausgeht, dass Platon aufgrund der Abschaffung der Privatfamilie "gezwungen" war, die Gleichstellung der Geschlechter einzuführen, formuliert sie später, mit Verweis auf die Kritik von William Jacobs, vorsichtiger und spricht davon, dass die Auflösung der Familie zur Gleichstellung der Geschlechter geführt habe. Vgl. Okin 1992, 38, Vgl. auch Bluestone (1987), $104 f$. 
gründen somit, folgt man Okins Platoninterpretation, die eingeschränkten öffentlichen Partizipationsmöglichkeiten von Frauen. Nicht emanzipatorische, sondern funktionalistisch theoretische Erwägungen seien demnach durchweg bestimmend für die Stellung der Frauen in Platons Werken. Den Thesen von Okin haben insbesondere William Jacobs (1978) und Nicholas D. Smith (1980) zu Recht entgegengehalten, dass auf die Abschaffung der Privatfamilie rein logisch keineswegs die Gleichstellung der Frauen im Wächterstand folgen müsse. In Anlehnung an Vlastos schließt Jacobs, dass nicht die Auflösung der Familie die Befreiung der Frauen bedinge, sondern umgekehrt, die gleichen sozialen Funktionen von Frauen und Männern im Wächterstand eine Auflösung der Familie nahe legten. (Jacobs 1978, 29f). Für Smith lässt sich Platons Idee von der Gleichheit von Frauen und Männer im Wächterstand einzig damit begründen, dass dieser einer Minderheit von Frauen die Fähigkeit zur politischen Partizipation zugesprochen habe (N. D. Smith 1980,5f.).

Suchte sich Okin in ihren Überlegungen von der platonischen "Feminismusdebatte insofern zu distanzieren, als sie sich rein auf die Erklärung werkimmanenter Spannungen zwischen Politeia und Nomoi konzentrierte, so knüpft Arlene Saxonhouse explizit an die Debatte unter differenztheoretischer Perspektive an (Saxonhouse 1976, 196). Ausgehend von ganz ähnlichen Prämissen wie Monique Canto liegt ihr erklärtes Ziel darin, die Bedeutung von Frauen und Weiblichkeit innerhalb des klassischen politischen Denkens aufzuzeigen (Saxonhouse 1984, 7). Dabei wendet sie sich explizit gegen eine profeministische Lesart Platons. "Nevertheless, Plato has frequently become the darling of contemporary feminist theorists for his willingness to go against custom to suggest the possibilities of removing women from the private sphere and placing them in the public world." (Saxonhouse 1985, 46). Platons Zielsetzung sei keineswegs die Emanzipation der Frauen gewesen, sondern Frauen symbolisierten Platons Kritik am zeitgenössischen Politikverständnis. In Anlehnung an Allen Bloom (1968) vertritt Saxonhouse die These, dass Platon in der Politeia die Unrealisierbarkeit des idealen Staates vor allem anhand der Stellung der Frauen und der Einführung von Philosophenkönigen dokumentieren wollte. Die Idealstaatsidee der Politeia basiere auf der völligen Zerstörung der weiblichen Natur und bedürfe des völligen Ausschlusses des von Frauen repräsentierten Anderen, von Partikularität, Körperlichkeit und Begehren. "In order to create the uniformed city Socrates must destroy the female as female, the female as threat to the unity of human space." (Saxonhouse 1985, 17). Nur als "de-sexed and unnatural females" können Frauen im Wächterstand politisch zu Gleichen werden (Saxonhouse 1976, 196). Die "Pervertierung der Natur" werde damit zur Voraussetzung für die politische Partizipation von Frauen. Die Unmöglichkeit der Realisierbarkeit des Idealstaates zeige sich nicht nur an der Denaturalisierung des Weiblichen, sondern auch an der parallel dazu verlaufenden Denaturalisierung und Pervertierung der Philosophie. So steht in Saxonhouse Platoninterpretation das Weibliche im Gegen- 
satz zum herrschenden, männlichen Politikverständnis, ebenso wie die Philosophie in Opposition zur praktischen Politik (Saxonhouse 1976, 202). Durch ihre Integration in den Bereich des Politischen werden die generativen Fähigkeiten des Weiblichen ebenso zerstört wie die der Philosophie. Die Parallelisierung, die Saxonhouse zwischen Weiblichkeit und Philosophie vornimmt, kulminiert in der dichotomen Gegenüberstellung von Geburt und Tod, Philosophie bzw. Weiblichkeit und Politik. »The activity of philosophy is a life-giving force, one that parallels the creation of life. The activity of politics is a death-directed power" (Saxonhouse 1985, 50). Im Vergleich zu den dem Weiblichen und der Philosophie zugewiesenen, "überlegenen" natürlich-generativen Fähigkeiten bleibt das Politische bestenfalls mangelhaft, eine "männliche "Antithese gegen die Natur. Diesen "philosophisch-weiblichen Schatten" habe Platon demonstrativ auf die Idealstaatsidee legen wollen, um damit die Unmöglichkeit idealer Politik bzw. die genuine Unvollkommenheit des Politischen zu illustrieren. Saxonhouse's Platoninterpretation ist aus einer radikal-differenztheoretischen Perspektive, bestimmt von einer Glorifizierung weiblicher Reproduktionsfahigkeiten. Ihre durchgängige Gleichsetzung von Weiblichkeit und Philosophie einerseits und Tod und Politik andererseits wird anhand der platonischen Primärquellen, darauf hat Natalie Bluestone hingewiesen, nicht schlüssig belegt (vgl. Bluestone 1987, 98ff. $)^{10}$, die Gleichheitsvorstellungen Platons werden insgesamt als "unnatürlich" und damit "unrealisierbar" abgetan. Für Natalie Bluestone, die selbst aus einer gleichheitsfeministischen Perspektive argumentiert, erscheint es deshalb fraglich, ob Saxonhouse Thesen überhaupt als "feministisch" bezeichnet werden können. In Bezug auf die von Saxonhouse konstatierte sexuell-reproduktiven weiblichen Überlegenheit führt sie aus: „She tells us that even women least skillful in this task do it better than men! I shudder how she would describe all those women today who either do not possess or do not exercise this skill in which their superiority supposedly lies" (Bluestone 1987, 100). Der hier noch zum Ausdruck kommende Konflikt über einen richtigen "feministischen Standpunkt" und eine "richtige" feministische Lesart der platonischen Texte, scheint sich bis Mitte der 90er Jahre endgültig beruhigt zu haben. In dem 1994 von Nancy Tuana herausgegebenen Kompendium zu "feministischen Platoninterpretationen" stehen die Überlegungen von Saxonhouse und Bluestone nebeneinander und dienen der Herausgeberin schlicht als Ausweis für die Varianz feministischer Perspektiven (Tuana 1994, 3ff.).

Ebenfalls differenztheoretisch argumentiert Jean Bethke Elshtain in ihrem bereits erwähnten, breit rezipierten Standardwerk "Public man and Private woman" gegen die These von der Gleichstellung der Frauen in Platons Wächterstand. Platon, und hier lehnt sich Elshtain an die Argumentation von Saxonhouse an ohne

10 Bluestone bezieht sich für diese Argumentation hauptsächlich auf die Dialolge Timaeus, und Symposion. 
diese jedoch zu erwähnen ${ }^{11}$, habe durch die völlige Deprivatisierung des Politischen im Wächterstand und die damit einhergehende Gleichstellung der Geschlechter vor allem von den Frauen einen unakzeptablen Preis gefordert: die Aufgabe ihrer weiblichen Identität. Wie für Saxonhouse, ist auch für Elshtain diese an Reproduktion, Mutterschaft und die daraus hervorgehende soziale Rolle der Frauen in der Familie gebunden. "Women suffer most heavily from Plato's denial of biological integrity as they can least escape biological imperatives" (Elshtain 1981, 38). Elshtains Platonkritik speist sich vor allem aus der normativen Prämisse essentialistischer Mütterlichkeit. ${ }^{12}$ Platons Idealstaatsvorstellungen in der Politeia erscheinen ihr dementsprechend scharf zu kritisieren: Platons Herangehensweise zeichnet sich durch "conceptual inadequacy" aus, seine Überlegungen zur Frauen- und Kindergemeinschaft deutet Elshtain schlicht als eine Überreaktion entstanden aus einer verzweifelten Angst vor Uneinigkeit und Desintegration (Elshtain 1981, 36f.).

Vergleicht man die unter gleichheits- und differenztheoretischen Prämissen vorgebrachten Argumente gegen eine profeministische Einordnung Platons, so liegt folgende Schlussfolgerung nahe: Aus beiden Perspektiven wird die funktionalistisch-instrumentelle Stellung der Frauen in Platons staatsphilosophischen Werken als zentrales Gegenargument hervorgehoben. Während die am Gleichheitsbegriff orientierten Interpretinnen damit den Begriff "feministisch" für Platons politische Theorie bereits für entkräftet halten, bedarf die unter differenztheoretischen Perspektiven vollzogene Ablehnung darüber hinaus noch einer weiteren, essentialistisch begründeten Untermauerung. Diese beinhaltet neben einem Absehen von der - nicht nur zeithistorischen - Besonderheit der platonischen Ausführungen zur Geschlechtergleichheit, eine ahistorische und kontextunabhängige Objektivierung von weiblicher Identität. Insofern sagen die jeweils zugrunde gelegten Weiblichkeitskonzepte mehr über das Feminismusverständnis der Platoninterpretinnen aus, als über die widersprüchlichen Aussagen Platons zu Gleichheit und Differenzen zwischen den Geschlechtern (vgl. auch Föllinger $1996,56){ }^{13}$

11 Elshtain stellt überhaupt kaum Bezüge zur feministischen Platonforschung her, einzige in den Fußnoten erwähnte Ausnahmen sind Okin und Rosenthal, vgl. dies 1981, 19ff.

12 Elshtain fordert eine Reformulierung des Öffentlichen und Privaten auf der Grundlage weiblicher Identität. Familie und Mutterschaft erscheinen ihr als zentrale Erfahrungsbereiche, da sie universal und transhistorisch existentiell für die Entwicklung einer menschlichen und sozialen Existenz seien, vgl. dies. 1981, 302ff.

$13 \mathrm{Vgl}$. hierzu auch die Kritik von Judith Butler an der differenztheoretischen Platoninterpretation von Luce Irigaray: „Denn schließlich ist Platons Szenographie der Intelligibilität auf den Ausschluss von Frauen, Sklaven, Kindern und Tieren angewiesen. (...) Irigaray hilft der Sache nicht weiter, weil sie es unterlässt, die metonymische Verbindung zwischen Frauen und diesen Anderen ganz herzustellen, während sie das 'Anderswor als das Weibliche idealisiert und sich aneignet. Aber was ist dieses 'Anderswor des Irigarayschen 'Anderswor? Wenn das Weibliche nicht die einzige oder primäre Sichtweise ist, die aus der Ökonomie männlicher Vernunft ausgeschlossen ist, was wird dann und wer wird dann im Zuge der Irigarayschen Analyse ausgeschlossen?" (Butler 1995, 75ff.). 


\section{Dekonstruktionen feministischer Platondeutungen}

Spätestens ab Ende der 80er Jahre verliert die Debatte um »Platons Feminismus» an rhetorischer Schärfe und analytischer Bedeutung. Im Zuge des Paradigmenwechsels von der Frauen- zur Geschlechterforschung gewinnt der kritische Rückblick auf die bisherigen feministischen Platonforschungen zunehmend an Gewicht. Neuere Platonforschungen konstituieren sich unter dem Schlagwort der "Dekonstruktion von Geschlecht" vornehmlich in Abgrenzung zu "älteren" feministischen Lesarten. In einer Replik auf die Ausführungen von Arlene Saxonhouse verbindet Wendy Brown ihre Zielsetzung einer Dekonstruktion maskulinistischen Geschlechterkonstruktionen mit einer expliziten Kritik am Stand der bisherigen feministischen Platonforschungen. "While the political thought of Plato has generated substantial amount of feminist commentary in recent years, discussion of Plato's treatment of gender has been mostly limited to his explicit utterances of women and his program for "equal opportunity" put forth in Book V of the Republic (Brown 1988, 595). Genauere Angaben, auf welche Autorinnen bzw. Quellen sich ihre Kritik bezieht, bleibt Brown allerdings komplett schuldig. ${ }^{14}$ Ihren Fokus legt sie auf die in Platons Werk zum Ausdruck kommende Kritik an den herrschenden Konzeptionen und Praktiken "politischer Männlichkeit“. Platon habe keineswegs, wie von Arlene Saxonhouse nahegelegt, die Opposition von "weiblicher" Philosophie und "männlicher" Politik zeigen wollen, seine Ausführungen zielten vielmehr auf die Kritik konventioneller, männlicher Rationalitäts- und Politikvorstellungen. Die geschlechtsspezifischen Überlegungen dienten ihm dabei rein als subversives Instrument, um die herrschenden Annahmen über Männlichkeit und Politik grundlegend in Frage zu stellen (Brown 1988, 599).

Obwohl Brown zu Recht für die feministische Platonforschung die Ausweitung der Frageperspektive auf das Geschlechterverhältnis und die in den politischen Theorien zum Ausdruck gebrachten Männlichkeitskonzeptionen anmahnt, bleibt ihre Konstruktion des feministischen Debattenverlaufes holzschnittartig, die Verankerung ihrer Thesen in der feministischen Platonforschung unzureichend. $^{15}$

Eine ähnlich reduktive Sicht auf die bisherigen feministischen Platonforschungen findet sich auch für die unter dekonstruktiven Vorzeichen formulierte Kritik von Elisabeth Spelman. So zeige, Spelman zufolge, die feministische Platonforschung sehr eindrücklich, welche biases auch unter feministischen Vorzeichen produziert worden seien, ohne dies auch nur wissenschaftlich transparent

14 Einzig Arlene Saxonhouse und Mary O'Brien werden von Brown in den Fußnoten positiv hervorgehoben, vgl. Brown 1988, IX.

15 Sie selbst verbindet meines Erachtens die funktionalistischen Interpretationen der Geschlechtergleichheit, wie sie von Okin und Annas vorgelegt worden sind, mit den Thesen von Saxonhouse. 
zu machen, oder als problematisch anzusehen. Es müsse als traurige Ironie aufgefasst werden, dass Platon immer wieder als »erster feministischer Philosoph« beschrieben worden sei (Spelman 1988,19). Mit Blick auf die bisherigen feministischen Klassikerforschungen zu Platon und Aristoteles kommt sie zu einem durchweg ernüchternden Ergebnis, allerdings ebenfalls ohne die Autorinnen zu benennen, auf die sich ihre Grundsatzkritik bezieht. "«I would be more enthusiastic about leaving Plato and Aristotle on the library shelfs (...) if I had not come to believe that too many of the critiques of their inherent sexism are equally exclusionary in their focus and concern." (Spelman 1988,6). Der eingeschränkte "middle class bias« feministischen Denkens präge, laut Spelman, auch die feministische Platonforschung, wobei sie die bereits seit Ende der 70er Jahre vorliegenden Kritiken (u.a. von Lange und Annas) an genau diesen Einschränkungen feministischer Untersuchungsperspektiven konsequent unerwähnt lässt.

\section{Fazit}

Welche Folgerungen legt diese Analyse der bisherigen feministischen Platonforschungen nahe?

Erstens die feministisch ausgewiesenen Platonbilder erweisen sich selbst dann als kontrovers, wenn die AutorInnen von ganz ähnlichen theoretischen Prämissen ausgehen. So kann weder von einem positiven Platonbild unter gleichheitstheoretischen Vorzeichen, noch von der Konstruktion eines gegenteiligen Bildes unter differenztheoretischer Perspektive gesprochen werden. Die innerhalb der feministischen Platonrezeption bis heute vorherrschende dualistische Einteilung in profeministisch und antifeministisch argumentierende AutorInnen kann dementsprechend kaum Erklärungswert zugewiesen werden.

Darüber hinaus lässt sich zweitens keine chronologische Dramaturgie von anfänglich profeministischen hin zu späteren antifeministischen Lesarten Platons konstruieren. Die "Feminismusthese" stieß von Anfang an auf heftigen Widerstand, erweist sich jedoch gleichzeitig bis heute als konstitutive Referenzgröße feministischer Platonforschungen (vgl. auch Bluestone 1988, 41ff.).

Drittens ist die These von einer feministischen Komplettverwerfung platonischer politischer Philosophie ebenso abzulehnen, wie die These von der Notwendigkeit einer Komplettrevision feministischer Platonforschung unter den Vorzeichen dekonstruktiver Kritik. Die in Platons Werk vorherrschenden Differenzen zwischen Frauen und Männern und innerhalb der weiblichen und männlichen Genusgruppen sind auch in der älteren feministischen Platonforschung immer wieder angedeutet, auf das elitäre Politikverständnis Platons verwiesen worden. Wenn »jüngere" feministische Autorinnen dies nicht zur Kenntnis nehmen, so kann ihre wissenschaftliche Arbeitsweise als ahistorisch oder ihr eigener wissenschaftlicher Originalitätsanspruch als allzu weit ausgedehnt kriti- 
siert werden. Jedenfalls produzieren sie damit, ganz gegen die eigene theoretische Verortung, selbst Ausschlussannahmen, die sich als nicht haltbar erweisen.

Die feministischen Kontroversen über die politische Philosophie Platons unterstreichen viertens die gerade in jüngster Zeit wieder explizit vertretene These, dass politische Ideengeschichtsschreibung ihre produktive Spannung nicht aus einer "gelassenen Bestandsaufnahme" gewinnt, sondern aus der notwendigen Konfrontation klassischer Texte mit aktuellen Problemstellungen und Theoriedebatten (Münkler 2003, 115; vgl. auch Asbach 2002, 658). Vor diesem Hintergrund erscheint der fortgesetzte Ausschluss feministischer Perspektiven aus dem ideengeschichtlichen Fachdiskurs und das nahezu durchgängig zu beobachtende Nebeneinander von "feministischer" und "mainstream" Ideengeschichtsschreibung wissenschaftlich nicht begründbar.

Feministische Platonforschungen spiegeln insgesamt eindrücklich Theoriekonjunkturen wider, die jeweils ganz unterschiedliche Fragestellungen und Fokussierungen - die Gleichstellung von Frauen und Männern, die Suche nach der unterdrückten Weiblichkeit, die Frage nach der weiblichen Andersheit, die Analyse hegemonialer Männlichkeitskonzepte oder die Suche nach Differenzen - hervorbringen und die nicht zuletzt auch darüber entscheiden, welche Thesen und Ergebnisse der Klassikerforschungen im feministischen Diskurs tradiert werden und welche eben nicht. Der zu Recht beklagte Ausschluss bzw. die Nichtrezeption feministischer Analyseperspektiven innerhalb des ideengeschichtlichen mainstream findet hierin eine abgeschwächte - allerdings wissenschaftlich nicht besser begründbare - feministische Entsprechung.

\section{Literaturverzeichnis}

Allen, Christine Garside. (1975): Plato on Women. in: Feminist Studies. Jg. 2, H. 2-3, S. 131-138.

Allen, Prudence R. S. M. (1985): The Concept of Woman. The Aristotelian Revolution 750 BC-AD 1250. Montreal.

Annas, Julia (1976): Plato's Republic and Feminism. in: Philosophy. Jg. 51, S. 307-321.

Asbach, Olaf (2002): Von der Geschichte politischer Ideen zur "History of Political Discourse«? Skinner, Pocock und die "Cambridge School«. in: Zeitschrift für Politikwissenschaft. Jg. 12, H. 2/02, S. 637-667.

Benhabib, Seyla/Nicholson, Linda (1987): Politische Philosophie und die Frauenfrage. in: Pipers Handbuch der politischen Ideen, Hrsg. von Iring Fetscher und Herfried Münkler. Bd. 5:Vom Zeitalter des Imperialismus bis zu den neuen sozialen Bewegungen. München, S. 513-562.

Bloom, Allen (1968): The Republic of Plato. Translated with Notes and an Interpretative Essay. 2. Auflage. New York.

Bluestone, Natalie Harris (1987): Women in the Ideal Society. Plato's Republic and the Modern Myths of Gender. Oxford.

Brown, Wendy (1988): "Supposing truth were a woman." Plato's suberversion of masculine discourse. in: Political Theory 16/4, S. 594-616.

Butler, Judith (1995): Körper von Gewicht: Die diskursiven Grenzen des Geschlechts. Frankfurt am Main. 
Canto, Monique (1986): The Politics of Women's Bodies: Reflections on Plato. in: Suleiman, Susan Rubin (Hrsg.): The Female Body in Western Culture. Contemporary Perspectives. Cambridge, S. 339-353.

Cohen, David (1987): The legal status and political role of women in Plato's laws. in: Revue internationale des droits de l'antiquite. Ser. 3 (34), S. 27-40.

Doyé, Sabine/Heinz, Marion/Kuster, Friederike (Hrsg.) (2002): Philosophische Geschlechtertheorien. Ausgewählte Texte von der Antike bis zur Gegenwart mit Kommentar und Bibliographie. Stuttgart.

Elshtain, Jean Bethke (1981): Public Man and Private Woman. Women in the Social and Political Thought. Princeton.

Föllinger, Sabine (1996): Differenz und Gleichheit. Das Geschlechterverhältnis in der Sicht griechischer Philosophen des 4. bis 1. Jahrhunderts v. Chr. Stuttgart.

Fortenbaugh, W.W. (1975): On Plato's Feminism. in: Apeiron. Jg. 9, S. 1-4.

Heinz, Marion (1997): Das metaphysische Fundament der Geschlechterordnung in den Staatsidealen von Platon und Aristoteles. in:Völger, Gisela: Sie und Er. Frauenmacht und Männerherrschaft. Geschlechterbeziehungen im Kulturvergleich. Bd. 1, Köln, S. 99-108.

Heinz, Marion (2002): Gleichheit der Natur von Mann und Frau: Platon. in: Doyé, Sabine/Heinz, Marion/Kuster, Friederike (Hg.): Philosophische Geschlechtertheorien. Ausgewählte Texte von der Antike bis zur Gegenwart. Stuttgart, S. 67-71.

Höffe, Otfried (Hrsg.) (1997): Platon. Politeia. Berlin.

Jacobs, William (1978): Plato on Female Emancipation. in: Apeiron. Jg. 12, H.1, S. 29-31.

Kelsen, Hans (1942): Platonic Love. in: The American Imago. A Psychoanalytical Journal for the Arts and Sciences, 3/1-2, S.3-110.

Kersting, Wolfgang (1999): Platons Staat. Darmstadt.

Kraut, Richard (1997) (Hrsg.): Plato's Republic. Critical Essays. Lanham.

Lange, Linda (1979): The Function of Equal Education in Plato's Republic and Laws. in: Clark, Lorenne M. G./Lange, Linda (Hrsg.): The Sexism of Social and Political Theory. Toronto, S. 4-15.

Martin, Jane Roland (1985): Reclaiming a Conversation. The Ideal of Educated Women. New Haven.

Münkler, Herfried (2003): Politische Ideengeschichte. in: Ders. (Hrsg.): Politikwissenschaft. Ein Grundkurs. Reinbek bei Hamburg.

Okin, Susan Moller (1992, erstmals 1979): Women in Western Political Thought. 2. Auflage, Princeton.

Okin, Susan Moller (1977): Philosopher Queen and Private Wives: Plato on Women and the Family. in: Philosophy and Public Affairs 6/4, S. 345-369.

Ottmann, Henning (2001): Geschichte des politischen Denkens. Bd.1/2:Von Platon bis zum Hellenismus. Stuttgart.

Osborne, Martha Lee (1975): Plato's Unchanging View of Woman: A Denial That Anatomy Spells Destiny. in: The Philosophical Forum, Summer 1975, S. 447-452.

Pierce, Christine (1994): Eros and Epistemology. in: Bar On, Bat-Ami (Hrsg.): Critical Feminist Readings in Plato and Aristotle. New York, S. 25-39.

Pierce, Christine (1973): Equality: Republic V. in: The Monist 57/1, S. 1-11.

Platon: Der Staat. Übersetzt und erläutert von Otto Appelt. Hamburg ${ }^{11} 1989$.

Pomeroy, Sarah B. (1974): Feminism in BookV of Plato's Republic. in: Apeiron 8/1, S. 33-35.

Rauschenbach, Brigitte (1998): Politische Philosophie und Geschlechterordnung. Eine Einführung. Frankfurt am Main.

Rosenthal, Abigail (1973): Feminism Without Contradictions. in: The Monist 57/1, S. 28-42.

Rousseau, Jean Jacques: Emil oder über die Erziehung. In neuer dt. Fassung von Ludwig Schmidts. Paderborn ${ }^{11} 1993$.

Saxonhouse, Arlene W. (1976): The Philosopher and the Female in the Political Thought of Plato. in: Political Theory 4, S. 195-212. 
Smith, Janet Farrell (1994): Plato, Irony, and Equality. in: Tuana, Nancy (1994) (Hrsg.): Feminist Interpretations of Plato. University Park, PA, S. 25-48.

Smith, Nicolas (1980):The Logic of Plato's Feminism. in: Journal of Social Philosophy 11, S. 5-11.

Smith, Nicholas D. (1983): Plato and Aristotle on the nature of woman. in: Journal of the History of Philosophy 21/4, S. 467-478.

Spelman, Elisabeth V. (1988): Inessetial Woman. Problems of Exclusion in Feminist Thought. London.

Tuana, Nancy (1994) (Hrsg.): Feminist Interpretations of Plato. University Park, Pennsylvania.

Vlastos, Gregory (1997, zuerst 1989): Was Plato a Feminist? in: Kraut, Richard (1997) (Hrsg.): Plato's Republic. Critical Essays. Lanham, S. 115-128.

Ward, Julie K. (1996) (Hrsg.): Feminism and Ancient Philosophy. New York.

Wender, Dorothea (1973): Misogynist, Paedophile, and Feminist. in: Arethusa 1, S. 75-90.

Zehnpfennig, Barbara (2001): Platon zur Einführung. Hamburg. 